\title{
Immune privilege or privileged immunity?
}

\author{
JV Forrester ${ }^{1}, \mathrm{H} \mathrm{Xu}^{1}, \mathrm{~T} \mathrm{Lambe}^{2}$ and R Cornall ${ }^{2}$
}

Immune privilege is a concept that has come of age. Where previously it was considered to be a passive phenomenon restricted to certain specialized tissues, it is now viewed as comprising several mechanisms, both active and passive, shared in many aspects with emerging notions of the mechanisms of peripheral tolerance. The relative degrees of immune privilege vary from tissue to tissue depending on the number and strength of each of the mechanisms contained in that tissue. Immune privilege can be generated in non-privileged sites such as the skin and allografts, and is a property of the tissue itself. We therefore propose that, in addition to canonical central and peripheral tolerance mechanisms, there is a third route whereby the organism promotes self-antigen non-reactivity centered on the specific properties of each tissue and varying accordingly (relative degrees of immune privilege). This third mechanism of inducing immunological tolerance, as it is a local tissue phenomenon, might have particular therapeutic significance, for instance in devising strategies for induction of immunity to tumors by disrupting immune privilege or in preventing graft rejection by promoting immune privilege.

\section{INTRODUCTION}

The notion of immune privilege emerged from studies of a phenomenon that had been recognized for over 130 years (if Neiderkorn's historical analysis is to be accepted ${ }^{1}$ ), namely that allografts, which were rapidly rejected from tissues such as the skin, were accepted when placed in certain other sites, particularly the brain and anterior chamber of the eye. Privilege (which by definition means some form of advantage) was interpreted to be conferred on these grafts in that they did not evoke an immunological rejection reaction and were "allowed" by the organism to survive. As further investigations were conducted, it became clear that many other sites demonstrated "privilege" and the notion developed that there were both "privileged sites" and "privileged tissues". 2 Furthermore, an additional phenomenon became evident through experimentation on animal models, namely that privilege or at least a modified form of immunological responsiveness (immune deviation) was transferable to other sites in an antigen-specific manner following inoculation of antigen into a privileged site, such as the eye and brain. ${ }^{3,4}$ When the eye was the site of inoculation, this phenomenon was termed anterior chamber-associated immune deviation (ACAID) ${ }^{5}$

More recently, the concept of immune privilege has been extended to many other forms of modified immune responses, in particular to the "impaired" immune response to tumors and to the acceptance of grafts in classically non-privileged sites such as the skin and heart induced through immunological tolerizing procedures. $^{6,7}$

However, since the time of the first description, it was rapidly recognized that the most universal form of immune privilege occurs at the fetomaternal interface. The mechanisms underpinning this form of immune privilege have been extensively reviewed. ${ }^{8-11}$

\section{THE SEMANTICS OF PRIVILEGE}

It is worthwhile to consider briefly what is meant by privilege. According to the Oxford English Dictionary, privilege can be described as an advantage, right, or benefit that is not available to everyone; a form of exemption. Even Shakespeare used the word in the context of a "sanctuary," a term that Mellor and Munn apply to T-regulatory cells as "single cell sanctuaries." 12 The self-evident advantage of immune privilege to the organism is that the collateral damage generated during a "normal" immune response is so attenuated that non-renewable tissues (e.g., retina, brain) are protected; however, the corollary is that the target of attack (e.g., the foreign antigen, see Figure 1 toxoplasma antigen) is not removed and becomes a Trojan horse, waiting for the moment when privilege is lost and overwhelming tissue damage occurs. Caspi ${ }^{13}$ has recently highlighted this conundrum in suggesting that immunological privilege of many tissues is maintained at a cost or risk to the organ when the tissues are breached; thus, self-antigens in "privileged" organs,

${ }^{1}$ Department of Ophthalmology, University of Aberdeen, Aberdeen, Scotland. ${ }^{2}$ Department of Medicine, University of Oxford, Oxford, England. Correspondence: JV Forrester (j.forrester@abdn.ac.uk)

Received 16 May 2008; accepted 2 June 2008; published online 2 July 2008. doi:10.1038/mi.2008.27 
unlike self antigens in "non-privileged" organs, which normally induce a tolerizing response, actually are viewed as non-self or foreign antigens since they have not been "seen" by the developing immune system (immunological ignorance). This might actually be a less-than-perfect form of tolerance and, in many ways, is another way of describing sequestration, as originally propounded by Medawar. ${ }^{14}$

\section{BLOOD-TISSUE BARRIERS AND IMMUNE PRIVILEGE}

The notion of sequestration of antigen as the backdrop to immune privilege developed as a result of the historical, anatomical, and physiological studies revealing the barrier to the passage of molecules, particularly dyes, which occurred in tissues such as the brain and retina (for review, see ref. ${ }^{15}$ ). There is little

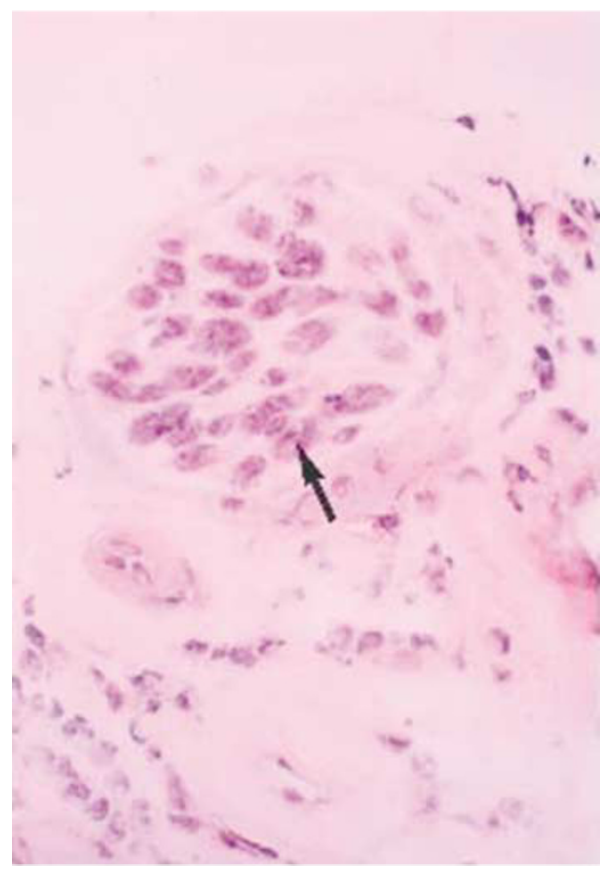

Figure 1 Toxoplasma cyst residing in uninflamed human retina. doubt that in the normal central nervous system (CNS) tissues, there is a substantial barrier to the passage of molecules, which can be precisely defined in terms of molecular size. ${ }^{16}$ However, further examination reveals that the integrity of this barrier in the CNS is variable depending on the vessel type (artery, capillary, or vein) and its pericellular composition. It was assumed that this barrier would also be present to large particles such as cells, but clearly this could not be so, as cell-tracking studies have revealed that there is a constant turnover of "resident" bone marrow-derived cells to the brain ${ }^{17}$ and retina; ${ }^{18}$ in addition, circulating $\mathrm{T}$ cells and comigrating myeloid cells can penetrate the CNS tissues prior to the onset of autoimmune inflammatory disease. ${ }^{19-21}$ However, this is not simply an open door to the passage of leukocytes: activated, but not naive ("resting"), T cells can penetrate the blood-retinal barrier and do not do so immediately they enter the circulation but only after a period of several hours, during which time the vasculature upregulates adhesion molecules and other receptors. ${ }^{21,22}$ In contrast, only fresh recent myeloid emigrants from the bone marrow are able to cross the blood-retinal barrier whereas "activated" macrophages do not ${ }^{23}$ (Figure 2). Thus, although the blood-CNS barrier appears to be strictly controlled to the passive movement of molecules into the tissues, the passage of cells seems to be more selective and to involve active transendothelial migratory processes.

A second barrier proposed as an explanation of CNS immune privilege was the absence of lymphatics and antigen-presenting cells (APCs) in the target tissue; however, recent studies have confirmed earlier literature that there is a substantial, lymphatic drainage connecting the meninges and ventricular system, if not the brain parenchyma, directly through the cribriform plate to the deep cervical lymph nodes, ${ }^{24}$ whereas the eye contains rich networks of $\mathrm{APCs}^{25,26}$ and has a functioning lymphatic system through the submandibular lymph node connecting all parts of the eye except the retina proper. ${ }^{27,28}$ The potential to rapidly develop lymphatics, which are abundant in inflamed ocular tissues, ${ }^{29}$ may be due to the presence of precursor cells in many ocular tissues. ${ }^{30}$
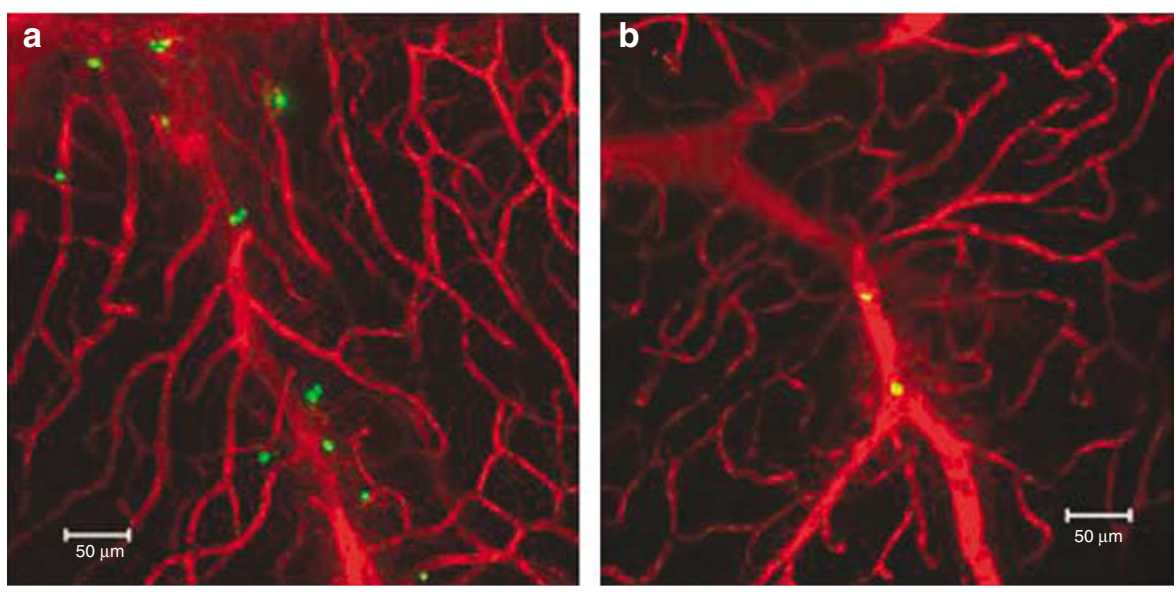

Figure 2 Transendothelial migration of monocytes in inflamed retina. Fresh BM cells (a) or in vitro cultured BM macrophages (b) were adoptively transferred into day $18 \mathrm{EAU}$ mice. Mice were killed $48 \mathrm{~h}$ later and retinal whole mounts were prepared for confocal microscopy. Only fresh monocytes were able to cross the endothelial barrier and enter the tissue. BM, bone marrow; EAU, autoimmune uveoretinitis. 


\section{HIERARCHY OF “IMMUNE PRIVILEGE” AND SUMMARY OF MECHANISMS INVOKED}

As originally proposed, immunological "privilege" was restricted to certain tissues such as the brain and the eye. In addition, Medawar was intrigued at the survival of the fetus in which clearly the host had to contend with non-self, paternal antigens. It became clear, however, that privilege could be extended to many tissues including the testis, ${ }^{31}$ liver, ${ }^{32}$ hair follicle, ${ }^{33}$ and mucosa. ${ }^{34,35}$ Even unexpected tissues, such as the gut, ${ }^{36}$ tumors, ${ }^{37}$ skin, and lymph node itself have been included as immune-privileged sites under certain circumstances (see below). Most recently, strategies to promote the acceptance of grafts in sites not normally associated with privilege, such as the heart and skin, have been described in terms of induced immunological privilege. ${ }^{6,7}$ As most of these organs do not have impermeable blood-tissue barriers, other mechanisms are required to explain how "privilege" operates under these circumstances. In one sense, the idea that blood-tissue barriers did not fully explain the phenomena of immune privilege dates back to the early work of Medawar, who demonstrated that allogeneic skin grafts that were not rejected after being grafted into the anterior chamber of the eye were also subsequently accepted when removed from the eye and regrafted into the skin. ${ }^{38}$ This protection from rejection was antigen-specific as it did not extend to third-party skin grafts. Thus, some form of antigen-specific immune suppression appeared to be actively induced such that systemic immunological protection against that antigen was generated after implantation of allografts into the eye.

Active mechanisms that might contribute to immunological privilege extend in many circumstances to the tissues themselves. Thus, neurological tissues express many molecules that regulate T-cell function including CD200, ${ }^{39} \mathrm{FasL},{ }^{40-43}$ and TRAIL $^{44-46}$ (for review, see ref. ${ }^{44}$ ). Ligation of some of these molecules may, however, act like a double-edged sword: for instance, under certain circumstances, Fas-FasL interactions can promote inflammation and tissue damage, ${ }^{47,48}$ whereas in other contexts, activated T-cell apoptosis is induced. ${ }^{41,49}$ This duality of function resonates well with the idea of a cost-benefit implication for immune privilege. ${ }^{13}$ The testis, which incidentally has an extensive mature lymphatic system, ${ }^{50}$ also demonstrates several tissue-associated immunoregulatory molecules such as FasL and TRAIL, ${ }^{51}$ and its resident parenchymal cells release a range of anti-inflammatory cytokines important in the induction of T-regulatory cells.

Active "privilege-mimicking" mechanisms can extend to tissues not normally associated with privilege, such as exposed tissue surfaces, which are the major routes of initial foreign antigen entry. ${ }^{52}$ For instance, the gastric mucosa has recently been shown to demonstrate "privilege" during Helicobacter infection through Fas-FasL interactions. ${ }^{52}$ The ocular surface, unlike the subconjunctival space, ${ }^{53}$ has similar properties to other mucosal surfaces and expresses many active immunosuppressive mechanisms including the secretion of short-range local mediators ${ }^{35}$ as well as participating in the more general mucosa-associated lymphoid tissue downregulatory mechanisms. ${ }^{54}$ The oral mucosa has been viewed similarly. ${ }^{55}$ The gastrointestinal tract has also been included as a tissue that under certain circumstances can express privilege: indeed, under normal homeostatic conditions, the mechanisms that render the gut flora and dietary antigens non-immunogenic are considered by some to be part of the "immune privilege" process and are sustained through a range of mechanisms, such as the secretion of immunosuppressive mediators, which ensure the generation of tolerogenic APCs, inflammatory anergic macrophages, and various sets of regulatory $\mathrm{T}$ cells that promote immunological non-responsiveness. ${ }^{36}$ Clearly, the distinction between immune privilege and immunological tolerance becomes blurred when the phenomena are discussed in these terms.

Tissues, such as the liver, have long been recognized to be immunologically privileged and, indeed, are one of the frequently used explanations for relative success with liver transplants. ${ }^{32}$ The locus of immune privilege in the liver has been attributed to a little-known anatomical feature i.e., the space of Disse (for review, see ref. ${ }^{56}$ ). However, there are many other well-recognized active mechanisms. For instance, there are many cell-surface immunoregulatory molecules expressed by liver cells, such as FasL and TRAIL as well as many secreted molecules including transforming growth factor- $\beta$ (TGFb), interleukin-10, and other mediators. In addition, liver-derived dendritic cells (DCs) have been shown to be tolerogenic and immune-deviating compared to mature bone marrow-derived DCs, ${ }^{57}$ in a similar way to the proposal for eye-derived APCs (see below). An alternative and topical mechanism suggested is through the enzyme indoleamine oxidase, which converts tryptophan to kynurenine intermediates, which in turn have significant downregulatory effects by inducing antigen-specific T-regulatory cells. This is considered to be one of the central mechanisms underpinning the capabilities of "tolerizing DCs" (see below). Other theories abound regarding the mechanisms of liver-centered immune privilege. For instance, Crispe et al. ${ }^{31}$ have recently proposed an interesting hypothesis based on the continuous exposure of liver cells to gut flora-derived endotoxin, which deviates liver cells to produce a set of T-cell tolerizing cytokines, antigen-presenting cell-surface molecules, and inhibitory costimulation. T-regulatory cells are thus generated in large numbers rather than T-cell anergy or immune deviation as seen in ACAID. ${ }^{32}$

Alopecia areata is an autoimmune disease in which the target for immune attack is the hair follicle. This structure was proposed by Westgate et al.$^{58}$ to possess immune privilege in which special emphasis was placed on active mechanisms involving limited major histocompatibility complex (MHC) class Ia expression by hair follicle epithelial cells and suppression of natural killer cell and macrophage activity. ${ }^{59,60}$ Low expression of MHC class I appears to be linked to secretion of immunoregulators such as TGFb and interferon-g, similar in some ways to the anterior chamber of the eye. Interestingly, Fas-FasL mechanisms were not involved. ${ }^{61}$

Surprisingly, in some ways, the secondary lymphoid tissues, normally the seat of induction of immunity, are now regarded, if not as immune-privileged sites, at least as the source of cells 
that are implicated in immune privilege. They are a major site of residence of peripheral CD4 $+\mathrm{CD} 25+\mathrm{T}$-regulatory cells, they can direct Th2-based mucosal immune tolerance, ${ }^{62}$ and the spleen appears in some obscure way to be the center of a multicellular immune response, involving natural killer-1. $1 \mathrm{~T}$ cells, B cells, F4/80 macrophages, and $\gamma \delta$-T cells, which results in the generation of CD8 + T-suppressor cells mediating ACAID. ${ }^{5,63-69}$

The site par excellence demonstrating immune privilege is the feto-maternal interface of the placenta. Clearly, there is extensive interchange of molecules and cells between the mother and the fetus, and therefore blood-tissue barriers, apart from some special examples of selective transport and even secretion, do not play a major role. Instead, active mechanisms are important and include apoptosis induced by FasL ${ }^{70}$ and TRAIL, ${ }^{45} \mathrm{NK}$ cells, specific molecules such as $\mathrm{MIF}^{71}$ and the non-conventional MHC Class I HLA G $\left({ }^{8,72,73}\right)$, and several sets of T regulatory cells linked in some cases to high levels of IDO. ${ }^{74}$ Interestingly, expression of HLA G has been linked to some forms of human melanomas ${ }^{75}$ (see next paragraph).

Although this section of the review cannot describe all the known sites of immune privilege, two remaining sites of importance merit discussion: solid tumors and sites of chronic infection, ${ }^{12}$ particularly the skin. Although tumor-related molecules, such as corticotrophin-related hormone in ovarian tumors, and FasL more generally, ${ }^{76}$ have been implicated in prevention of the antitumor immune response, ${ }^{77}$ it is now recognized that survival of tumors depends in large part on suppression of autologous antitumor immunity, e.g. by downregulating MHC class I antigens and Fas while upregulating FasL. Anti-tumor immune reactivity is also expressed in many cases by high levels of tumor-infiltrating regulatory $\mathrm{T}$ cells. ${ }^{78}$ Some tumors promote T-regulatory cells and immune deviation by secretion of molecules such as galectin- $1^{79}$ or TGFb. ${ }^{80}$ In addition, they frequently downregulate MHC class I antigens and Fas while upregulating FasL. Even the tumor-draining lymph node may be involved in this process and generate tumor antigen-specific T-regulatory cells. ${ }^{81}$ Expression of CCR 4 by CD $4+\mathrm{CD} 25+\mathrm{T}$ regs in certain tumors appears to be linked with the development of tumorassociated immune privilege. ${ }^{82}$

Finally, one persisting puzzle for investigators of inflammatory disease is how chronic infections develop and persist. Recent studies in skin Leishmania have shown that, in fact, chronic infection is generated by persistence of the parasite. This is achieved by subvention of the immune response through mediators secreted by the parasite, which modify the function of infiltrating macrophages and DCs. Peters et al.${ }^{83}$ have shown that this is assisted in Leishmania-infected mice by the large numbers of steady-state, naturally occurring CD4 + CD25+ T-regulatory cells mediated through interleukin- 10 secretion. In one sense, this is linked to well-recognized methods of immune evasion that are employed by many infectious agents, particularly latent viruses, which induce T-regulatory cells at the site of infection, as for instance herpes simplex virus induction of CD8 + regulatory $\mathrm{T}$ cell in the trigeminal ganglion after initial corneal epithelial cell infection. ${ }^{84}$ This is an ambivalent situation, as escape from latency is easily achieved, resulting in recrudescence of herpes simplex keratitis. ${ }^{84}$ The precarious nature of this process is highlighted by the persistence of Human Immunodeficiency Virus in the CNS, in part promoted by the immune-privileged state of this tissue. ${ }^{85}$

\section{IS IMMUNOLOGICAL TOLERANCE TO "PRIVILEGED" ANTIGENS MODIFIED?}

The notion of "privileged" self-antigens residing in certain tissue raises the difficult question of whether immunological tolerance to such "privileged" antigens is as effective or complete as tolerance to antigens in non-privileged sites. Tolerance to selfantigens is achieved through two main mechanisms: central tolerance in the thymus in which deletion of self-reactive cells is the dominant mechanism and peripheral tolerance in which the few autoreactive cells that have escaped central tolerance are regulated. Peripheral tolerance is mediated by a range of mechanisms including deletion (after activation), anergy, and suppression by regulatory $\mathrm{T}$ cells. ${ }^{86}$

Induction of central tolerance is mediated by thymic expression and presentation of peripheral antigens by medullary thymic epithelial cells, cortical epithelial cells, and DCs, and it is partly under the control of the autoimmune regulator (AIRE) gene. ${ }^{87,88}$ For some retinal (presumed privileged) antigens, expression levels of antigen by medullary thymic epithelial cells appeared to correlate with resistance to autoimmune inflammation. ${ }^{89}$ In addition, although AIRE-deficient mice develop autoimmune disease to several tissue-specific antigens, loss of expression of a single retinal antigen, interphotoreceptor retinalbinding protein (IRBP), abrogated autoimmune inflammation in the eye but not in other AIRE-regulated tissues. In addition, absence of IRBP in the thymus renders mice susceptible to retinal inflammation, all of which support a significant role for control of ocular inflammation in this immune-privileged site through central tolerance mechanisms. ${ }^{90}$

It would appear, therefore, that tolerance to privileged antigens, in the context of the retina at least, is under the control of a range of mechanisms, but it is difficult to quantify and correlate levels of tolerance vs. susceptibility to autoimmunity irrespective of whether the antigen resides in a privileged organ or in a presumed non-privileged site. For instance, does the level of medullary thymic epithelial cell expression of a particular antigen in the thymus determine whether tolerance to that antigen is effective, and does that correlate directly or inversely with the degree of immunological ignorance (sequestration) that a "privileged" antigen enjoys?

\section{SEQUESTRATION AND IMMUNOLOGICAL IGNORANCE}

We have recently attempted to address this question of whether there is a correlation between systemic immunological tolerance and "privileged" vs. (presumptive) "non-privileged" antigens. For this, we constructed two transgenic models in which a neo(pseudo-self)-antigen is expressed in the target cells. The foreign antigen hen egg lysozyme (HEL) was expressed in two cell types: (a) the melanocyte, which is broadly distributed in many tissues and is presumably a source of "non-privileged" 

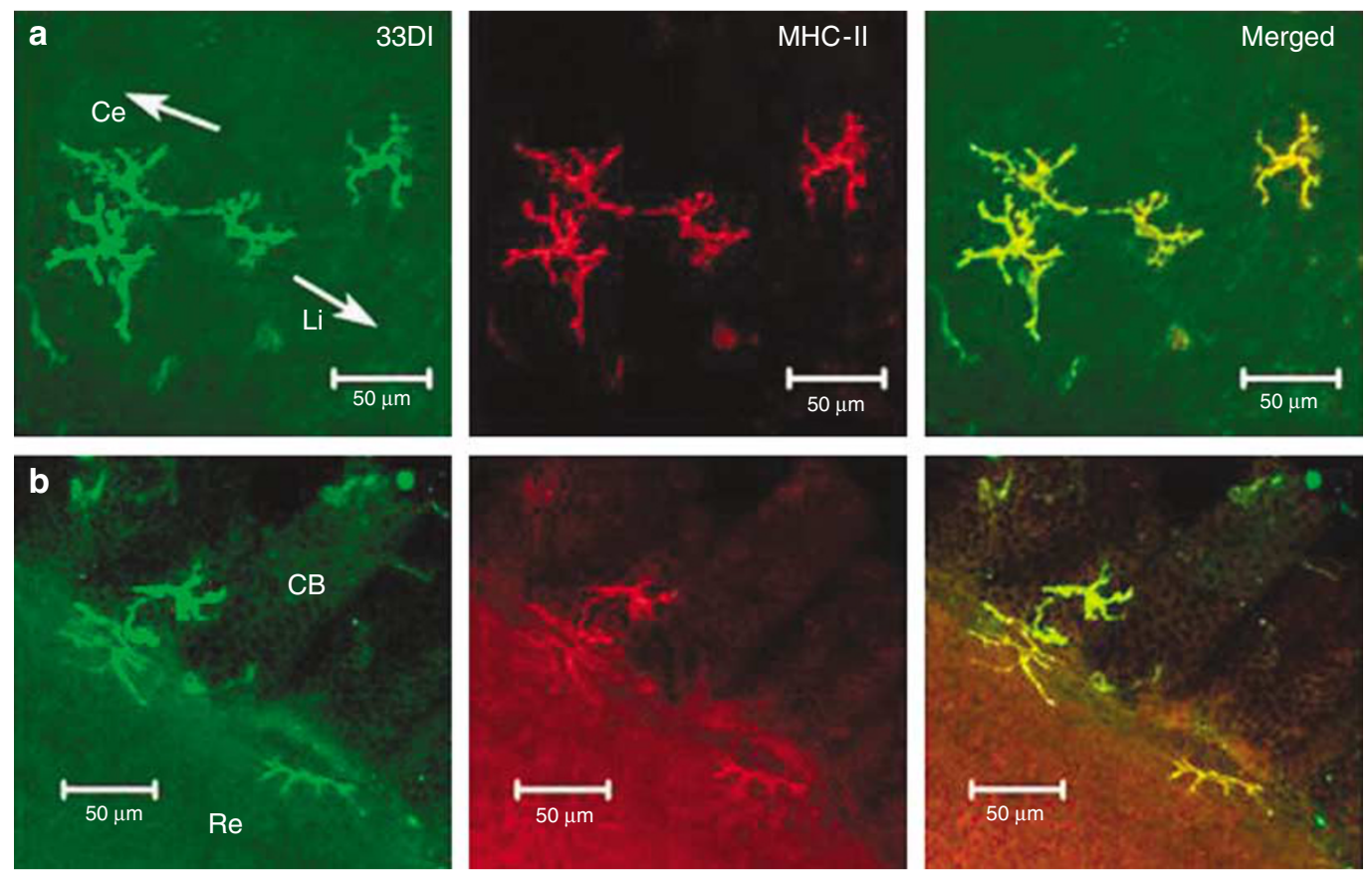

Figure 3 33D1+MHC-II + cells in normal mouse eye. (a) 33D1+MHC-II+ cells in peripheral corneal tissue. Ce, central cornea; Li, limbus. (b) 33D1+MHC-II+ cells in peripheral retinal tissue. CB, ciliary body; Re, Retina.

antigen; and (b) the photoreceptor cell in the retina, in which many retina-specific, presumed "privileged" antigens reside. ${ }^{91,92}$ HEL was expressed in the melanocyte under the control of the promoter for tyrosinase-related protein-2 $(\operatorname{Trp} 2)^{93}$ and in the retina under the control of the promoter for IRBP, ${ }^{94}$ and it was also membrane-associated (i.e., not secreted) through linkage to the MHC class I promoter. In both cases, the single transgenic mice were normal, showing no signs of disease. However, when the mice were crossed with a transgenic mouse, in which $70 \%$ of the CD4 + T cells expressed a T-cell receptor for the immunodominant peptide 46-61 of the HEL protein, both strains of mice developed autoimmune disease. In the case of the doubletransgenic HEL-Trp2:TCR mice, a spontaneous form of vitiligo developed, ${ }^{93}$ whereas in the case of the double-transgenic HEL-IRBP:TCR mice, spontaneous autoimmune uveoretinitis developed. ${ }^{94}$ Investigation of disease induction in each model, however, revealed different underlying mechanisms of disease. In the vitiligo mice, T cell-mediated melanocyte killing via Fasl, but not perforin, was required to permit disease, whereas in the case of the autoimmune uveoretinitis mice, there appeared to be a defect in peripheral tolerance through limited anergy induction. There was also a possible defect in T-regulatory cells, but further studies are required to elucidate this. However, there was a clear absence of Fas-FasL and perforin-mediated mechanism in the autoimmune uveoretinitis mice. ${ }^{94}$

These studies hint at the possibility that "privileged" antigens are relatively ignored by the immune system (old-fashioned sequestration), at least during development of peripheral tolerance mechanisms, even though there is clear evidence of some level of central tolerance induction through expression of antigen and regulatory control through Aire in the thymus, as crossing the (double-transgenic) HEL-IRBP:TCR mice to the Aire knockout mice led to an acceleration of disease, indicating that central tolerance through Aire was functional. The possibility that the "privileged" tissue may provide the mechanisms for immunological ignorance and exert local control over the immune response adds another dimension to the notion of tolerance (see below).

\section{PRIVILEGE IS RELATIVE AND CONTEXT-DEPENDENT}

Immune privilege, as originally conceived, has gone through many drafts of its mechanistic hypotheses. The initial concept of a blood-tissue barrier proposed by Medawar ${ }^{14,38}$ stemmed from his observations that grafts in the anterior chamber of the eye were accepted only if they did not adhere to the iris/uveal tissue and develop a vascular communication. This notion fell into disfavor as "privilege" and intact tissue barriers could not be universally demonstrated especially for tissues such as the placenta. It was replaced by the idea that impaired immune responses in privileged tissues resulted from a defective lymphatic circulation and, even more importantly, from an absence of APCs capable of local induction of the immune response. The model tissue for this was par excellence the cornea, but even this tissue has been found to contain many passenger leukocytes and even precursor DCs, ${ }^{95}$ whereas the brain and the eye were found to contain large networks of APCs, if not in the parenchymal tissues, at least in the coverings and adjacent tissues. ${ }^{25,96,97}$ In this context, the recent identification of a population of MHC class II-positive 33D1 cells in the periphery of the retina and cornea has revealed a potential route for T-cell activation in these privileged sites (Figure 3) ${ }^{98} 33 \mathrm{D} 1+$ DCs are a subset of MHC class $\mathrm{II}^{\mathrm{hi}}$-expressing DCs found in specialized regions in the spleen 
and are thought to be important in promoting active adaptive immunity. ${ }^{99}$ However, their position in the marginal regions of the eye suggests that they might also have a role in immune tolerance mediated by DCs. ${ }^{100}$ In other privileged tissues such as the testis, it was easier to discount the lymphatic vessel deficiency theory as a general explanation for privilege.

In this context, the role of DCs in mediating tolerance has now become established dogma. The current paradigm, in fact, suggests that the major role of bone marrow-derived tissue-surveying DCs is to promote peripheral tolerance and prevent reactivity to self-antigens. In this scenario, 'tolerizing' DCs capture self-antigen released from the tissues during normal cell turnover (housekeeping apoptosis) and migrate to the draining lymph node where they present the antigen to $\mathrm{T}$ cells. Stochastic presentation to an autoreactive $\mathrm{T}$ cell fails to lead to pathogenic activation of the $\mathrm{T}$ cells because presentation occurs in the absence of costimulation, for which the DCs would have had to be exposed to innate immune triggers such as Tolllike receptor ligands or other stimuli. Several other mechanisms have been proposed for DC mediation of peripheral tolerance, including the induction of T-regulatory cells. This might occur through release of suppressive cytokines such as interleukin-10 and TGFb, or through the expression of immunosuppressive ligands such as FasL and PDL1 or -2. A well-defined mechanism by which DCs induce peripheral T-regulatory cell is through indoleamine oxidase, which as indicated above is important in the catabolism of tryptophan. The kynenurine products have the capacity to promote T-regulatory cells directly, and indoleamine oxidase-expressing DCs, particularly plasmacytoid DCs, have been identified in immune-privileged tissues such as the cornea. Other potential DC-related enzyme systems promoting immune suppression are the arginase and inducible nitric oxide synthase routes. Arginase leads to nitric oxide and peroxynitrite production, whereas release of nitric oxide in certain contexts has a direct immunosuppressive effect at least in vitro. ${ }^{101,102}$

As vascular/tissue barriers failed to fulfil criteria for defining privilege and lack of cells capable of presenting antigen locally in the tissue also fell short of the mark as an explanation, attention moved to active regulatory mechanisms. For many years, ocular immune privilege has been associated with the phenomenon of ACAID (see above), and brain-associated immune deviation has also been demonstrated. ${ }^{4}$ ACAID to alloantigens has been more difficult to demonstrate but there is evidence that in mice in which corneal allografts are not rejected, the systemic immune response to specific minor antigens is reduced on delayed hypersensitivity testing. ${ }^{103}$ ACAID is mediated through T-regulatory cells (predominantly CD8 + T cells, among other cells including $\gamma \delta$-T cells, ${ }^{68} \mathrm{CD} 4 \mathrm{~T}$ cells, and B cells, mostly generated in the spleen) ${ }^{104}$ and undoubtedly demonstrates the development of antigen-specific modulation of the immune response. Indeed, inoculation of antigen in the anterior chamber of the eye has been shown to reduce the severity of lung inflammation in an experimental model of asthma. ${ }^{105}$ Teleologically, ACAID and presumptive (but not formally demonstrated) retina-associated immune deviation have been proposed to function as immunological brakes through moderating the systemic immune response to rare tissue antigens, which, not having been seen previously by the developing immune system and thus prevented from inducing regular tolerance, might through injury or infection be exposed to the adult immune system and set off a typical foreign antigen response. Indeed, this is one of the concepts put forward to explain the infrequency of the ocular condition, sympathetic ophthalmia, a disorder in which the uninjured eye develops spontaneous inflammation after penetrating injury to the first eye. ${ }^{106}$

There is an issue, however, as to whether ACAID amounts to immune privilege. ACAID is mediated by T-regulatory cells as indicated above. Central to both induction of the adaptive immune response ( $\mathrm{T}$ effector cells) and the expansion of T-regulatory cells is antigen presentation and specificity of the responses: direct evidence for the latter in the context of privilege has been difficult to demonstrate. ${ }^{94}$ Privilege, as proposed in its original form, might be better restricted to the concept of protection of the tissue by the tissue from the collateral damage of a full-blown immune response. For many years, an expanding list of factors associated with the local expression of immune privilege has been enumerated (see Table 1) and include soluble factors such as neuropeptides and TGFb as well as cell-associated factors such as neuronal CD200, HLA G, and Qa-1.

\section{CAN NON-PRIVILEGED SITES BE CONVERTEDTO PRIVILEGED SITES?}

The surprising findings of inducible immune privilege in the skin, as part of chronic infection, indicate that even the most immunoreactive tissues can be made relatively immunosilent. A relative degree of immune privilege can now be attributed to many tissues, and especially to new tissue antigens (tumor antigens and fetal antigens), fueling considerable interest in attempts to induce immune privilege for therapeutic purposes: this applies both to inducing immune privilege in tissues such as allografts to prevent their rejection and to breaking privilege, for instance, in tumors to promote their rejection. Cobbold and Waldmann ${ }^{6,7,107}$ have been pioneers of the concept of infectious tolerance in which tolerance induced in one grafted individual by CD4T cell depletion and other immunological manipulations can be transferred to another through T-cell transfer i.e., the second individual will accept a graft from the original donor without additional manipulation. Moreover, the second recipient will also accept a graft in time from a third unrelated individual, indicating that tolerance can spread to unrelated antigens in an "infectious" manner. ${ }^{6}$ Most recent studies of this phenomenon have indicated that, in fact, the grafts are colonized by T-regulatory cells, which as effector cells are known to be non-antigenspecific (i.e., although induced in response to specific antigen, they have a broad-spectrum immunoregulatory function against many T-cell specificities). ${ }^{108}$ The immunosuppressive effect, therefore, appears to reside in the graft and is lost when the graft is depleted of T-regulatory cells. The parenchyma of the graft releases immunosuppressive mediators such as TGFb, ${ }^{107}$ which promote T-regulatory cell expansion locally in the graft and inhibit the function of the alloreactive $\mathrm{T}$ cells. 
Table 1 Factors in the eye and ocular tissues that modify the immune response

\begin{tabular}{|c|c|c|c|c|c|}
\hline Type of factor & Example & T-cell function & $\begin{array}{l}\text { Macrophage } \\
\text { activity }\end{array}$ & $\begin{array}{l}\text { Antigen } \\
\text { presentation }\end{array}$ & $\begin{array}{l}\text { Cell killing } \\
\text { apoptosis }\end{array}$ \\
\hline \multirow[t]{2}{*}{ Neuropeptide } & $\begin{array}{l}\text { VIP, PACAP CGRP } \\
\text { Melanocortin }\end{array}$ & Inhibits & Inhibits & & \\
\hline & Substances P & & Promotes & & \\
\hline Neurotransmitter & GABA, glycine & Inhibits & & & \\
\hline Cytokine & TGFb, IL10 & & & & \\
\hline Membrane ligand & $\begin{array}{l}\text { FasL, CD200 TRAIL, } \\
\text { APRIL, }\end{array}$ & & & & Promotes \\
\hline Membrane receptor & TLRs & ?Inhibits & Promotes & & \\
\hline Cellular enzymes & IDO, L-arginase & Inhibits & & Inhibits & Promotes \\
\hline MHC protein & Qa-1 & & & Inhibits & \\
\hline Cannabinoids & & Inhibits & & ?Inhibits & ?Promotes \\
\hline Free radical & Peroxynitrate & & Promotes & Inhibits & Promotes \\
\hline
\end{tabular}

Abbreviation: MHC, major histocompatibility complex.

ascavenger activity?.

\section{TOLERANCE VS. PRIVILEGE}

The concepts of tolerance and immune privilege have matured along similar but parallel pathways over more than half a century. The notion of tolerance arose pari passu with adaptive immunity from the evidence that not all antigens induce an immune response, that antigens varied in their immunogenicity, and that in any case there was the problem of self/non-self discrimination. ${ }^{109}$ Despite newer variations on the theme that have incorporated responses of a non-adaptive nature (the danger hypothesis ${ }^{110}$ and the discovery of less-exquisitely selective innate immune receptors responding to foreign antigens ${ }^{111}$ ), the ideas of tolerance and immunity to antigens form the basis of immunological dogma. Meanwhile, immune privilege has been viewed until recently as an interesting curiosity related to specific tissues or sites in the organism, which were exceptions or refinements proving the rules of the larger concepts of tolerance and immunity. It was only as evidence emerged that immune privilege was not a purely passive process, that the phenomenon of ACAID involved generation of T-regulatory cells, and that the tissues generally could moderate immune responses merely by their characteristic properties that immune privilege came into the mainstream of immunological thought once more. ${ }^{12}$

The question really reduces to this: is immune privilege a distinct phenomenon or is it simply another form of tolerance? Tolerance has been defined in terms of non-reactivity to selfantigens, underpinned by such notions as the danger hypothesis $^{113}$ and linking of adaptive immunity to innate immunity, ${ }^{111}$ and is generally seen as a mechanism of tightly regulating immune responses to foreign antigens, thus preventing massive self-antigen-induced destructive disease. In contrast, immune privilege is viewed as a tissue-specific mechanism designed to prevent excessive damage to vital organs while allowing some level of immune response to proceed. Simpson ${ }^{112}$ has suggested that there is in fact a large element of overlap between privilege and tolerance, particularly in the induction of regulatory $\mathrm{T}$ cells, whereas Caspi ${ }^{13}$ has suggested that in fact immune privilege based on immunological ignorance (see above) is a risky strategy for the organism, as organ-specific peripheral tolerance mechanisms are imperfectly developed and leave the tissue exposed to damage from antigen-specific $\mathrm{T}$ cells that have escaped central deletion.

Mellor and Munn ${ }^{12}$ adhere to the concept of immune privilege as a distinct phenomenon on the basis that tolerance, both peripheral and central, is a systemic processes mediated by circulating or at least trafficking cells involved in immune surveillance, while privilege is a local phenomenon, acting locally to prevent effector cell damage and involving the conversion of Th0 cells to resident in situ T-regulatory cells through secretion of tissue-produced mediators such as TGFb. It is possible that such factors are actually produced by tissue-resident DCs (see above) or by parenchymal cells themselves.

They further suggest that tissues such as the mucosal lining of the gut display strong evidence of privilege or tissuecentered immunosuppressive properties as the gut has to handle a large micro-organismal load, whereas tissues such as the eye and the brain are in contrast more susceptible to the damaging effects of inflammation (if it occurs in the tissue) as they rarely encounter microorganisms. This echoes the high cost-benefit ratio of immune privilege in these classical sites as propounded by Caspi. ${ }^{13}$ This idea fits with the effects of inflammation in the intraocular compartments and also with recent evidence from allografts, where the immune privilege is restricted to the local site of the graft and is not detectable elsewhere. However, there is the problem of ACAID or antigen-specific systemic immune 
deviation: theoretically, it would be possible to induce immunological non-responsiveness or, at a minimum, immunological deviation by "tolerising" the organism through intraocular inoculation of antigen and subsequently challenging the organism to the same antigen. Experimentally, this has been demonstrated in a model of allergic lung disease. ${ }^{104}$ On the face of it, the mechanics of this are little different from mucosal tolerance protocols. Similar attempts to induce tolerance have been made through other immune-privileged routes such as the testis ${ }^{50,114,115}$ and the conjunctiva of the eye. ${ }^{54}$

\section{IS IMMUNE PRIVILEGE A USEFUL CONCEPT?}

As there is so much overlap in the mechanisms of immunological tolerance, particularly, mechanisms of peripheral tolerance and immune privilege, the question that must be asked is whether the concept of immune privilege adds anything to our understanding of immunity. In fact, immune privilege, as opposed to tissue-induced immune deviation, describes a property of the tissue and has value as it adds a third limb to our already well-developed concepts of tolerance mechanisms. Thus, there are two major cell-mediated mechanisms of systemic tolerance involving central mechanisms and peripheral mechanisms, in which trafficking cells survey the tissues for foreign antigen while suppressing or deleting cells with the potential to respond to self-antigen. These mechanisms are under genetic control, the complexity of which is increasingly being revealed. ${ }^{116-118}$ The third arm of tissue-based "local immune regulation" is another way of describing immune privilege and, as has been shown over many years, has a hierarchy that probably correlates to the strength or number of tissue-based regulatory mechanisms expressed by each tissue: thus, the retina and brain parenchyma possess many of the characteristics including pronounced blood-tissue barriers, relative absence of lymphatics, minimal numbers of APCs capable of presenting antigen in $s i t u$, and an extensive range of soluble and cell-bound inhibitors of the immune system, which under normal circumstances have the ability to promote induction of T-regulatory cells. It is also possible that some of these regulatory T cells are "resident" in normal uninflamed tissues, such as skin, and contribute to the overall immunosuppressive microenvironment. ${ }^{119}$ The properties and characteristics of each tissue may have a much greater influence over the immune response than we previously considered, and the tissue itself has been portrayed as a regulator of the response to "danger". ${ }^{120}$ This is new terminology for oldfashioned immune privilege.

Finally, it is also important to consider when and where immune privilege is established during development and if this has a bearing on whether antigens that are expressed early in development are more likely to induce central tolerance, compared to antigens that are expressed later in ontogeny, for which immunological ignorance may be the only route to prevent selfreactivity. For instance, some retinal antigens, e.g. neural antigens appear in the developing eye shortly after formation of the optic vesicle, whereas other antigens may not be fully expressed until after birth. This applies especially to the strongly autoantigenic photoreceptor antigens, which are not fully expressed in the retina until after birth, by which time the leaky hyaloid vascular system has given way to the formation of the anatomical and functional blood-retinal barrier, preventing access of membrane-bound antigens to the immune system. This issue has widespread implications and is best considered in the context of expression of "new" or "late" antigens, e.g. during puberty, in tumors and in the developing fetus. Tolerance to "late" selfantigens is currently explained by promiscuous gene expression in medullary thymic epithelial cells. ${ }^{121}$

\section{CONCLUSION}

We have reviewed the long-recognized concept of immune privilege in the context of the part it plays in the constant trade-off between tolerance vs. immunity or protection from "danger". ${ }^{110}$ We propose that rather than being an immunological curiosity attributable to certain specialized tissues such as the eye and the brain, all tissues have a relative degree of "privilege," allowing them to preserve varying levels of structural and functional integrity, depending on their intrinsic regenerative capacity, in the face of injury or danger. ${ }^{120}$ There are many mechanisms, both passive and active, underpinning "privilege" and probably many more to be discovered. We believe, therefore, that in addition to the two extensively described immune cell-based, central and peripheral, and innate and adaptive mechanisms of tolerance, this third tissue-based defense mechanism of protection against danger plays a major role in the survival of the organism. Sometimes, there is a $\cos ^{13}$ attached to this "privileged immunity" that may be too great, and the tissue, if not the organism, succumbs.

\section{REFERENCES}

1. Niederkorn, J.Y. See no evil, hear no evil, do no evil: the lessons of immune privilege. Nat. Immunol. 7, 354-359 (2006).

2. Streilein, J.W. Ocular immune privilege: the eye takes a dim but practical view of immunity and inflammation. J. Leukoc. Biol. 74, 179-185 (2003)

3. Galea, I., Bechmann, I. \& Perry, V.H. What is immune privilege (not)? Trends Immunol. 28, 12-18 (2007).

4. Wenkel, H., Streilein, J.W. \& Young, M.J. Systemic immune deviation in the brain that does not depend on the integrity of the blood-brain barrier. J. Immunol. 164, 5125-5131 (2000).

5. Niederkorn, J.Y. The induction of anterior chamber-associated immune deviation. Chem. Immunol. Allergy 92, 27-35 (2007).

6. Waldmann, H., Adams, E., Fairchild, P. \& Cobbold, S. Infectious tolerance and the long-term acceptance of transplanted tissue. Immunol. Rev. 212, 301-313 (2006).

7. Cobbold, S.P. et al. Immune privilege induced by regulatory T cells in transplantation tolerance. Immunol. Rev. 213, 239-255 (2006)

8. Hunt, J.S. Stranger in a strange land. Immunol. Rev. 213, 36-47 (2006).

9. Niederkorn, J.Y. \& Wang, S. Immune privilege of the eye and fetus: parallel universes? Transplantation 80, 1139-1144 (2005).

10. Kanellopoulos-Langevin, C., Caucheteux, S.M., Verbeke, P. \& Ojcius D.M. Tolerance of the fetus by the maternal immune system: role of inflammatory mediators at the feto-maternal interface. Reprod. Biol. Endocrinol. 1, 121 (2003).

11. Weetman, A.P. The immunology of pregnancy. Thyroid 9, 643-646 (1999).

12. Mellor, A.L. \& Munn, D.H. Creating immune privilege: active local suppression that benefits friends, but protects foes. Nat. Rev. 8, 74-80 (2008).

13. Caspi, R.R. Ocular autoimmunity: the price of privilege? Immunol. Rev. 213, 23-35 (2006).

14. Medawar, P.B. Immunological tolerance. Nature 189, 14-17 (1961). 
15. Bechmann, I., Galea, I. \& Perry, V.H. What is the blood-brain barrier (not)? Trends Immunol. 28, 5-11 (2007).

16. Matter, K. \& Balda, M.S. Holey barrier: claudins and the regulation of brain endothelial permeability. J. Cell Biol. 161, 459-460 (2003).

17. Chan, W.Y., Kohsaka, S. \& Rezaie, P. The origin and cell lineage of microglia: new concepts. Brain Res. Rev. 53, 344-354 (2007).

18. Xu, H., Chen, M., Mayer, E.J., Forrester, J.V. \& Dick, A.D. Turnover of resident retinal microglia in the normal adult mouse. Glia 55, 1189-1198 (2007).

19. Flugel, A. et al. Migratory activity and functional changes of green fluorescent effector cells before and during experimental autoimmune encephalomyelitis. Immunity 14, 547-560 (2001).

20. Xu, H., Manivannan, A., Liversidge, J., Sharp, P.F., Forrester, J.V. \& Crane, I.J. Requirements for passage of T lymphocytes across noninflamed retinal microvessels. J. Neuroimmunol. 142, 47-57 (2003).

21. Xu, H., Forrester, J.V., Liversidge, J. \& Crane, I.J. Leukocyte trafficking in experimental autoimmune uveitis: breakdown of blood-retinal barrier and upregulation of cellular adhesion molecules. Invest. Ophthalmol. Vis. Sci. 44, 226-234 (2003).

22. Xu, H., Manivannan, A., Crane, I.J., Dawson, R. \& Liversidge, J. Critical but divergent roles for $\mathrm{CD} 62 \mathrm{~L}$ and $\mathrm{CD} 44$ in directing blood monocyte trafficking in vivo during inflammation. Blood (2008) (e-pub ahead of print).

23. $\mathrm{Xu}, \mathrm{H}$. et al. Differentiation to the CCR2+ inflammatory phenotype in vivo is a constitutive, time-limited property of blood monocytes and is independent of local inflammatory mediators. J. Immunol. 175, 6915-6923 (2005)

24. Cserr, H.F. \& Knopf, P.M. Cervical lymphatics, the blood-brain barrier and the immunoreactivity of the brain: a new view. Immunol. Today 13 , 507-512 (1992).

25. McMenamin, P.G. Dendritic cells and macrophages in the uveal tract of the normal mouse eye. Br. J. Ophthalmol. 83, 598-604 (1999).

26. Forrester, J.V., McMenamin, P.G., Liversidge, J. \& Lumsden, L. Dendritic cells and "dendritic" macrophages in the uveal tract. Adv. Exp. Med. Biol. 329, 599-604 (1993).

27. Plskova, J., Holan, V., Filipec, M. \& Forrester, J.V. Lymph node removal enhances corneal graft survival in mice at high risk of rejection. $B M C$ Ophthalmol. 4, 3 (2004).

28. Plskova, J., Duncan, L., Holan, V., Filipec, M., Kraal, G. \& Forrester, J.V. The immune response to corneal allograft requires a site-specific draining lymph node. Transplantation 73, 210-215 (2002).

29. Dietrich, T. et al. Inhibition of inflammatory lymphangiogenesis by integrin alpha5 blockade. Am. J. Pathol. 171, 361-372 (2007).

30. Xu, H., Chen, M., Reid, D.M. \& Forrester, J.V. LYVE-1-positive macrophages are present in normal murine eyes. Invest. Ophthalmol. Vis. Sci. 48, 2162-2171 (2007).

31. Fijak, M. \& Meinhardt, A. The testis in immune privilege. Immunol. Rev. 213, 66-81 (2006).

32. Crispe, I.N., Giannandrea, M., Klein, I., John, B., Sampson, B. \& Wuensch, S. Cellular and molecular mechanisms of liver tolerance. Immunol. Rev. 213, 101-118 (2006).

33. Paus, R., Ito, N., Takigawa, M. \& Ito, T. The hair follicle and immune privilege. J. Investig. Dermatol. Symp. Proc. 8, 188-194 (2003).

34. Hickey, D.K. et al. Intranasal immunization with C. muridarum major outer membrane protein (MOMP) and cholera toxin elicits local production of neutralising IgA in the prostate. Vaccine 22 , 4306-4315 (2004).

35. Knop, E. \& Knop, N. Anatomy and immunology of the ocular surface. Chem. Immunol. Allergy 92, 36-49 (2007).

36. Iweala, O.I. \& Nagler, C.R. Immune privilege in the gut: the establishment and maintenance of non-responsiveness to dietary antigens and commensal flora. Immunol. Rev. 213, 82-100 (2006).

37. Benson, J.L. \& Niederkorn, J.Y. In situ suppression of delayed-type hypersensitivity: another mechanism for sustaining the immune privilege of the anterior chamber. Immunology 74, 153-159 (1991).

38. Medawar, P.B. Immunity to homologous grafted skin. Br. J. Exp. Pathol. 29, 58-69 (1948).

39. Copland, D.A. et al. Monoclonal antibody-mediated CD200 receptor signaling suppresses macrophage activation and tissue damage in experimental autoimmune uveoretinitis. Am. J. Pathol. 171, 580-588 (2007).

40. Askenasy, N., Yolcu, E.S., Yaniv, I. \& Shirwan, H. Induction of tolerance using Fas ligand: a double-edged immunomodulator. Blood 105, 1396-1404 (2005)
41. Dick, A.D. et al. Fas-Fas ligand-mediated apoptosis within aqueous during idiopathic acute anterior uveitis. Invest. Ophthalmol. Vis. Sci. 40, 2258-2267 (1999).

42. Ferguson, T.A. \& Griffith, T.S. A vision of cell death: insights into immune privilege. Immunol. Rev. 156, 167-184 (1997).

43. Ferguson, T.A. \& Griffith, T.S. A vision of cell death: Fas ligand and immune privilege 10 years later. Immunol. Rev. 213, 228-238 (2006).

44. Ferguson, T.A. \& Griffith, T.S. The role of Fas ligand and TNF-related apoptosis-inducing ligand (TRAIL) in the ocular immune response. Chem. Immunol. Allergy 92, 140-154 (2007).

45. Phillips, T.A. et al. TRAIL (Apo-2L) and TRAIL receptors in human placentas: implications for immune privilege. J. Immunol. 162, 6053-6059 (1999).

46. Wosik, K., Biernacki, K., Khouzam, M.P. \& Prat, A. Death receptor expression and function at the human blood brain barrier. J. Neurol. Sci. 259, 53-60 (2007).

47. Gregory, M.S. et al. A novel treatment for ocular tumors using membrane Fas $L$ vesicles to activate innate immunity and terminate immune privilege. Invest. Ophthalmol. Vis. Sci. 46, 2495-2502 (2005).

48. Gregory, M.S., Repp, A.C., Holhbaum, A.M., Saff, R.R., MarshakRothstein, A. \& Ksander, B.R. Membrane Fas ligand activates innate immunity and terminates ocular immune privilege. J. Immunol. 169, 2727-2735 (2002).

49. Griffith, T.S., Brunner, T., Fletcher, S.M., Green, D.R. \& Ferguson, T.A. Fas ligand-induced apoptosis as a mechanism of immune privilege. Science 270, 1189-1192 (1995).

50. Head, J.R., Neaves, W.B. \& Billingham, R.E. Reconsideration of the lymphatic drainage of the rat testis. Transplantation 35, 91-95 (1983).

51. Ferguson, T.A., Green, D.R. \& Griffith, T.S. Cell death and immune privilege. Int. Rev. Immunol. 21, 153-172 (2002).

52. Koyama, S. Apoptotic depletion of infiltrating mucosal lymphocytes associated with Fas ligand expression by Helicobacter pylori-infected gastric mucosal epithelium: human glandular stomach as a site of immune privilege. Dig. Dis. Sci. 45, 773-780 (2000).

53. Cousins, S.W., Trattler, W.B. \& Streilein, J.W. Immune privilege and suppression of immunogenic inflammation in the anterior chamber of the eye. Curr. Eye Res. 10, 287-297 (1991)

54. Gormley, P.D., Powell-Richards, A.O., Azuara-Blanco, A., Donoso, L.A \& Dua, H.S. Lymphocyte subsets in conjunctival mucosa-associatedlymphoid-tissue after exposure to retinal-S-antigen. Int. Ophthalmol. 22, 77-80 (1998).

55. Sugerman, P.B. et al. The pathogenesis of oral lichen planus. Crit. Rev. Oral. Biol. Med. 13, 350-365 (2002).

56. Geerts, A. History, heterogeneity, developmental biology, and functions of quiescent hepatic stellate cells. Semin. Liver Dis. 21, 311-335 (2001).

57. Khanna, A., Morelli, A.E., Zhong, C., Takayama, T., Lu, L. \& Thomson, A.W. Effects of liver-derived dendritic cell progenitors on Th1 - and Th2like cytokine responses in vitro and in vivo. J. Immunol. 164, 1346-1354 (2000).

58. Westgate, G.E., Craggs, R.I. \& Gibson, W.T. Immune privilege in hair growth. J. Invest. Dermatol. 97, 417-420 (1991).

59. Ito, T. et al. Maintenance of hair follicle immune privilege is linked to prevention of NK cell attack. J. Invest. Dermatol. 128, 1196-1206 (2008).

60. Ito, T., Ito, N., Bettermann, A., Tokura, Y., Takigawa, M. \& Paus, R. Collapse and restoration of MHC class-I-dependent immune privilege: exploiting the human hair follicle as a model. Am. J. Pathol. 164, 623-634 (2004).

61. Christoph, T. et al. The human hair follicle immune system: cellular composition and immune privilege. Br. J. Dermatol. 142, 862-873 (2000).

62. Alvarez, D., Arkinson, J.L., Sun, J., Fattouh, R., Walker, T. \& Jordana, M. Th2 differentiation in distinct lymph nodes influences the site of mucosal Th2 immune-inflammatory responses. J. Immunol. 179, 3287-3296 (2007).

63. Wilbanks, G.A. \& Streilein, J.W. Studies on the induction of anterior chamber-associated immune deviation (ACAID). 1. Evidence that an antigen-specific, ACAID-inducing, cell-associated signal exists in the peripheral blood. J. Immunol. 146, 2610-2617 (1991).

64. Wilbanks, G.A., Mammolenti, M. \& Streilein, J.W. Studies on the induction of anterior chamber-associated immune deviation (ACAID). III. Induction of ACAID depends upon intraocular transforming growth factor-beta. Eur. J. Immunol. 22, 165-173 (1992).

65. Takahashi, M. et al. Requirement for splenic CD4+ T cells in the immune privilege of the anterior chamber of the eye. Clin. Exp. Immunol. 116, 231-237 (1999). 
66. Stein-Streilein, J. \& Watte, C. Cross talk among cells promoting anterior chamber-associated immune deviation. Chem. Immunol. Allergy 92, 115-130 (2007).

67. Sonoda, K.H. \& Stein-Streilein, J. Ocular immune privilege and CD1d-reactive natural killer T cells. Cornea 21, S33-38 (2002).

68. Skelsey, M.E., Mellon, J. \& Niederkorn, J.Y. Gamma delta T cells are needed for ocular immune privilege and corneal graft survival. J. Immunol. 166, 4327-4333 (2001).

69. Niederkorn, J.Y. \& Mayhew, E. Role of splenic B cells in the immune privilege of the anterior chamber of the eye. Eur. J. Immunol. 25, 2783-2787 (1995).

70. Guller, S. Role of Fas ligand in conferring immune privilege to nonlymphoid cells. Ann. N. Y. Acad. Sci. 828, 268-272 (1997).

71. Arcuri, F. et al. Human decidual natural killer cells as a source and target of macrophage migration inhibitory factor. Reproduction 131, 175-182 (2006).

72. Menier, C., Riteau, B., Dausset, J., Carosella, E.D. \& Rouas-Freiss, N. HLA-G truncated isoforms can substitute for HLA-G1 in fetal survival. Hum. Immunol. 61, 1118-1125 (2000).

73. Hunt, J.S., Langat, D.K., McIntire, R.H. \& Morales, P.J. The role of HLA-G in human pregnancy. Reprod. Biol. Endocrinol. 4 (Suppl 1), S10 (2006).

74. Katz, J.B., Muller, A.J. \& Prendergast, G.C. Indoleamine 2,3dioxygenase in T-cell tolerance and tumoral immune escape. Immunol. Rev. 222, 206-221 (2008).

75. Cabestre, F.A. et al. HLA-G expression: immune privilege for tumour cells? Semin. Cancer Biol. 9, 27-36 (1999).

76. Linkermann, A., Qian, J., Lettau, M., Kabelitz, D. \& Janssen, O. Considering Fas ligand as a target for therapy. Expert Opin. Ther. Targets 9, 119-134 (2005).

77. Minas, $\mathrm{V}$. et al. Intratumoral $\mathrm{CRH}$ modulates immuno-escape of ovarian cancer cells through FasL regulation. Br. J. Cancer 97, 637-645 (2007).

78. Curiel, T.J. et al. Specific recruitment of regulatory T cells in ovarian carcinoma fosters immune privilege and predicts reduced survival. Nat. Med. 10, 942-949 (2004).

79. Juszczynski, P. et al. The AP1-dependent secretion of galectin-1 by Reed Sternberg cells fosters immune privilege in classical Hodgkin lymphoma. Proc. Natl Acad. Sci. USA 104, 13134-13139 (2007).

80. Wahl, S.M., Wen, J. \& Moutsopoulos, N. TGF-beta: a mobile purveyor of immune privilege. Immunol. Rev. 213, 213-227 (2006).

81. Munn, D.H. \& Mellor, A.L. The tumor-draining lymph node as an immune-privileged site. Immunol. Rev. 213, 146-158 (2006).

82. Ishida, T. et al. Specific recruitment of CC chemokine receptor 4-positive regulatory $T$ cells in Hodgkin lymphoma fosters immune privilege. Cancer Res. 66, 5716-5722 (2006).

83. Peters, N. \& Sacks, D. Immune privilege in sites of chronic infection: Leishmania and regulatory T cells. Immunol. Rev. 213, 159-179 (2006).

84. Lepisto, A.J., Frank, G.M. \& Hendricks, R.L. How herpes simplex virus type 1 rescinds corneal privilege. Chem. Immunol. Allergy 92, 203-212 (2007).

85. Persidsky, Y. \& Poluektova, L. Immune privilege and HIV-1 persistence in the CNS. Immunol. Rev. 213, 180-194 (2006).

86. Singh, N.J. \& Schwartz, R.H. Primer: mechanisms of immunologic tolerance. Nat. Clin. Pract. 2, 44-52 (2006).

87. Anderson, M.S., Venanzi, E.S., Chen, Z., Berzins, S.P., Benoist, C. \& Mathis, D. The cellular mechanism of Aire control of T cell tolerance. Immunity 23, 227-239 (2005).

88. Ferguson, B.J. et al. AIRE's CARD revealed, a new structure for central tolerance provokes transcriptional plasticity. J. Biol. Chem. 283, 1723-1731 (2008).

89. Egwuagu, C.E., Charukamnoetkanok, P. \& Gery, I. Thymic expression of autoantigens correlates with resistance to autoimmune disease. J. Immunol. 159, 3109-3112 (1997).

90. DeVoss, J. et al. Spontaneous autoimmunity prevented by thymic expression of a single self-antigen. J. Exp. Med. 203, 2727-2735 (2006).

91. Caspi, R.R. Immunopathology of the eye. Semin. Immunopathol. 30, 63-64 (2008).

92. Caspi, R.R. et al. Mouse models of experimental autoimmune uveitis. Ophthalmic. Res. 40, 169-174 (2008).

93. Lambe, T. et al. CD4T cell-dependent autoimmunity against a melanocyte neoantigen induces spontaneous vitiligo and depends upon Fas-Fas ligand interactions. J. Immunol. 177, 3055-3062 (2006).

94. Lambe, T. et al. Limited peripheral T cell anergy predisposes to retinal autoimmunity. J. Immunol. 178, 4276-4283 (2007).
95. Sosnova, M., Bradl, M. \& Forrester, J.V. CD34+ corneal stromal cells are bone marrow-derived and express hemopoietic stem cell markers. Stem Cells 23, 507-515 (2005).

96. McMenamin, P.G., Holthouse, I. \& Holt, P.G. Class II major histocompatibility complex (la) antigen-bearing dendritic cells within the iris and ciliary body of the rat eye: distribution, phenotype and relation to retinal microglia. Immunology 77, 385-393 (1992).

97. McMenamin, P.G. Distribution and phenotype of dendritic cells and resident tissue macrophages in the dura mater, leptomeninges, and choroid plexus of the rat brain as demonstrated in wholemount preparations. J. Comp. Neurol. 405, 553-562 (1999).

98. Xu, H., Dawson, R., Forrester, J.V. \& Liversidge, J. Identification of novel dendritic cell populations in normal mouse retina. Invest. Ophthalmol. Vis. Sci. 48, 1701-1710 (2007).

99. Dudziak, D. et al. Differential antigen processing by dendritic cell subsets in vivo. Science 315, 107-111 (2007).

100. Nussenzweig, M.C., Steinman, R.M., Witmer, M.D. \& Gutchinov, B. A monoclonal antibody specific for mouse dendritic cells. Proc. Natl Acad. Sci. USA 79, 161-165 (1982).

101. Hill, M., Zagani, R., Voisine, C., Usal, C. \& Anegon, I. Nitric oxide and indoleamine 2,3-dioxygenase mediate CTLA4lg-induced survival in heart allografts in rats. Transplantation 84, 1060-1063 (2007).

102. Rossner, S., Voigtlander, C., Wiethe, C., Hanig, J., Seifarth, C. \& Lutz, M.B. Myeloid dendritic cell precursors generated from bone marrow suppress $T$ cell responses via cell contact and nitric oxide production in vitro. Eur. J. Immunol. 35, 3533-3544 (2005).

103. Yamada, J. \& Streilein, J.W. Fate of orthotopic corneal allografts in C57BL/6 mice. Transpl. Immunol. 6, 161-168 (1998).

104. Zhang-Hoover, J. \& Stein-Streilein, J. Therapies based on principles of ocular immune privilege. Chem. Immunol. Allergy 92, 317-327 (2007).

105. Katagiri, K., Zhang-Hoover, J., Mo, J.S., Stein-Streilein, J. \& Streilein, J.W. Using tolerance induced via the anterior chamber of the eye to inhibit Th2-dependent pulmonary pathology. J. Immunol. 169, 84-89 (2002).

106. Forrester, J.V. Intermediate and posterior uveitis. Chem. Immunol. Allergy 92, 228-243 (2007)

107. Daley, S.R., Ma, J., Adams, E., Cobbold, S.P. \& Waldmann, H. A key role for TGF-beta signaling to T cells in the long-term acceptance of allografts. J. Immunol. 179, 3648-3654 (2007).

108. Yates, S.F. et al. Induction of regulatory T cells and dominant tolerance by dendritic cells incapable of full activation. J. Immunol. 179, 967-976 (2007).

109. Mitchison, N.A., Katz, D.R. \& Chain, B. Self/nonself discrimination among immunoregulatory (CD4) T cells. Semin. Immunol. 12, 179-183; discussion 257-344 (2000).

110. Matzinger, P. The danger model: a renewed sense of self. Science 296, 301-305 (2002).

111. Janeway, C.A. Jr. \& Medzhitov, R. Innate immune recognition. Annu. Rev. Immunol. 20, 197-216 (2002).

112. Simpson, E. A historical perspective on immunological privilege. Immunol. Rev. 213, 12-22 (2006).

113. Matzinger, P. An innate sense of danger. Ann. N. Y. Acad. Sci. 961, 341-342 (2002).

114. Head, J.R. \& Billingham, R.E. Immune privilege in the testis. II. Evaluation of potential local factors. Transplantation 40, 269-275 (1985).

115. Head, J.R., Neaves, W.B. \& Billingham, R.E. Immune privilege in the testis. I. Basic parameters of allograft survival. Transplantation 36 , 423-431 (1983).

116. Kersh, G.J. Transcriptional control of thymocyte positive selection. Immunol. Res. 29, 125-138 (2004).

117. Matsumoto, M. Transcriptional regulation in thymic epithelial cells for the establishment of self tolerance. Arch. Immunol. Ther. Exp. 55, 27-34 (2007).

118. Hollander, G., Gill, J., Zuklys, S., Iwanami, N., Liu, C. \& Takahama, Y. Cellular and molecular events during early thymus development. Immunol. Rev. 209, 28-46 (2006).

119. de Boer, O.J., van der Loos, C.M., Teeling, P., van der Wal, A.C. \& Teunissen, M.B. Immunohistochemical analysis of regulatory T cell markers FOXP3 and GITR on CD4+CD25+ T cells in normal skin and inflammatory dermatoses. J. Histochem. Cytochem. 55, 891-898 (2007)

120. Matzinger, P. Friendly and dangerous signals: is the tissue in control? Nat. Immunol. 8, 11-13 (2007).

121. Kyewski, B. \& Klein, L. A central role for central tolerance. Ann. Rev. Immunol. 24, 571-606 (2006). 